\title{
How to reduce circadian misalignment in rotating shift workers
}

\author{
This article was published in the following Dove Press journal: \\ ChronoPhysiology and Therapy \\ 13 July 2016 \\ Number of times this article has been viewed
}

\section{Charmane I Eastman \\ Biological Rhythms Research Laboratory, Behavioral Sciences Department, Rush University Medical Center, Chicago, IL, USA}

I have often thought that rapidly rotating shift work schedules that include night shifts should be abolished and replaced with fixed shifts. But maybe I was wrong, I used to think that there is no way to reduce the circadian misalignment between the master internal circadian clock (and thus all the circadian rhythms of the body) and the times for sleeping, working, and eating, because the circadian clock cannot reset (phase shift) fast enough to keep up with rapidly rotating shift work schedules. We know that circadian misalignment is largely responsible for the sleep deprivation that night shift workers suffer from, because their circadian clocks make it difficult to sleep during the day. ${ }^{1}$ Circadian misalignment is also responsible for the performance decrements and accidents during night shifts, when the body is geared up for sleep. ${ }^{2}$ Evidence continues to mount that shift work increases the risk for many chronic diseases, ${ }^{3,4}$ and it is classified as a "probable carcinogen" by the World Health Organization. ${ }^{5}$ The usual countermeasures and fatigue risk management plans for shift workers do not address the underlying problem, which is circadian misalignment. Instead, these plans entail symptomatic relief, such as the proper use of stimulants (like caffeine) and sleep aids, teaching basic sleep hygiene, and suggesting more days off for recovery after night work. I used to think that the only way to reduce circadian misalignment is to have permanent or fixed night work schedules, and then to trick the circadian clock into phase shifting to align with night work and day sleep, using appropriately timed artificial bright light and sleep (dark) schedules. ${ }^{3}$

There is a debate, which has been going on for decades, about whether fixed or rotating shift schedules are best. ${ }^{6-12}$ However, it is based on the fact that even the circadian rhythms of permanent night workers rarely adapt (phase shift) to adjust to their night work and day sleep schedules. ${ }^{13-15}$ The failure of circadian rhythms to adjust is attributed to the fact that night shift workers revert to sleeping at night on days off and are usually exposed to bright outdoor light on their way to home from the night shifts., 36 In other words, the light-dark cycle that most night shift workers are exposed to keeps their circadian clocks from delaying. In simulated night shift studies, however, we have reliably produced large phase delays of circadian rhythms, and thus improved night shift performance and alertness, by using bright light during the night shift, sunglasses on the way home, and prescribed, delayed, sleep (dark) schedules. ${ }^{17-20}$ As far as I know, this combination of interventions has not been adopted by real shift workers. I remain hopeful that public education about sleep and shift work will help promote this system for those who have fixed night shift schedules.
Correspondence: Charmane I Eastman Biological Rhythms Research Laboratory, I 645 West Jackson Boulevard, Suite 425, Chicago, IL 606I2, USA

$\mathrm{Tel}+\mathrm{I} 3 \mid 25634787$

Fax + I 3125634900

Email ceastman@rush.edu 
Recently, I have been thinking that there may also be hope for those afflicted with rapidly rotating shift work schedules. A worker who has to switch back and forth between 12-hour day shifts and 12-hour night shifts emailed me. He is working a rapidly rotating "DuPont-type" schedule, which is popular because it has 7 consecutive days off once every 4 weeks (Table 1). When shift workers in distress contact me, I tell them that it is not their fault that they have problems working night shifts and that it is normal to have trouble sleeping during the day and to feel like a zombie during and after working night shifts. I advise finding another job or to get transferred off of nights, perhaps with a letter from their doctor saying that they have shift work disorder. Although I do not like the term "shift work disorder", because it implies that there is something wrong with the individual, it does come in handy for doctor letters. The shift worker who contacted me impressed me with how much he had taught himself about circadian rhythms from reading difficult scientific papers like mine. He even discussed the body temperature minimum $\left(T_{\text {min }}\right)$ in his Emails. He had purchased a Re-Timer (Re-Time Pty Ltd, Adelaide, Australia), which are glasses designed to reset the circadian clock by shining green light into your eyes, but given his rotating work schedule they cannot help.

In trying to help him adjust to his impossible work schedule, I designed a new shift schedule (Table 2). Instead of the weekly changes between day shifts and night shifts, there are 2 weeks on days and 2 weeks on nights, and more days off are allotted for the transitions between these work shifts. Figure 1 shows this work schedule and the times for sleep (dark), bright light ( $\mathrm{S}$ and $\mathrm{L}$ ), melatonin $(\mathrm{M})$, and wearing sunglasses if outside (G) to gradually phase delay and phase advance the circadian clock back and forth between day shifts and night shifts to avoid circadian misalignment.

The goal is to keep the body temperature minimum $\left(T_{\min }\right)$, the sleepiest time of day (indicated by the triangles), within the sleep episodes (indicated by the horizontal lines; Figure 1). We estimate that the best sleep can be obtained when the $T_{\min }$ occurs between the beginning of sleep and 1 hour or 2 hours before the end of sleep. ${ }^{3,21}$ The $T_{\min }$ has three important functions. It is a marker for the time of the circadian clock, an estimate for the sleepiest time of day, and shows us when to use light or melatonin to phase shift the circadian clock. Light before the $T_{\text {min }}$ can help delay the circadian clock and light after the $T_{\min }$ can help advance the clock. $^{22-24}$ Therefore, to delay the circadian clock, we use bright light before the $T_{\text {min }}$ and block out or attenuate light after the $T_{\min }$. To advance, we use bright light after the $T_{\text {min }}$ and block out or attenuate light before the $T_{\min }$.

To help delay the circadian clock (to make it shift later) when shifts change from days to nights (days 4-9 in Figure 1), bright light from light boxes (L) is used before sleep and sunglasses $(G)$ are used if going outside soon after waking in the morning. Days 5-8 show good times for naps, which can make it easier to stay up later, but the specified times/duration are not rigid. The arrow heads pointing right on the sleep lines mean sleep as late as possible. The arrow tails pointing to the right mean going to sleep a little later is helpful. A very dark bedroom is important for successful delaying. The triangles show the expected delay of the circadian clock $(1 \mathrm{~h} / \mathrm{d})$. Some people may be able to delay more, $1.5 \mathrm{~h} / \mathrm{d}$ or even $2 \mathrm{~h} / \mathrm{d}$, with

Table I Current shift work schedule

\begin{tabular}{llllllll}
\hline Crew or week & Monday & Tuesday & Wednesday & Thursday & Friday & Saturday & Sunday \\
\hline I & D & D & D & D & X & X \\
2 & X & X & X & X & N & N & N \\
3 & N & X & X & X & D & D \\
4 & X & N & N & X & X & X \\
\hline
\end{tabular}

Notes: D, day shift; N, night shift; $X$, days off.

Table 2 Proposed shift work schedule

\begin{tabular}{llllllll}
\hline Crew or week & Monday & Tuesday & Wednesday & Thursday & Friday & Saturday & Sunday \\
\hline I & D & X & D & D & X & X & X \\
2 & X & N & X & X & N & N \\
3 & N & X & N & N & X & X \\
4 & X & D & X & X & D & D & D \\
\hline
\end{tabular}

Notes: The schedules are designed for four crews working 12-hour shifts. For each day, one crew works nights, one crew works days, and two crews are off. All workers rotate through the 4 weeks and then repeat the 4-week sequence. Each week has the usual billing cycle of Monday through Sunday. Thus, each week has three or four 12 hour work shifts, which fulfills the payroll (overtime) constraints of many companies. D, day shift; N, night shift; $X$, days off. 
TIME OF DAY

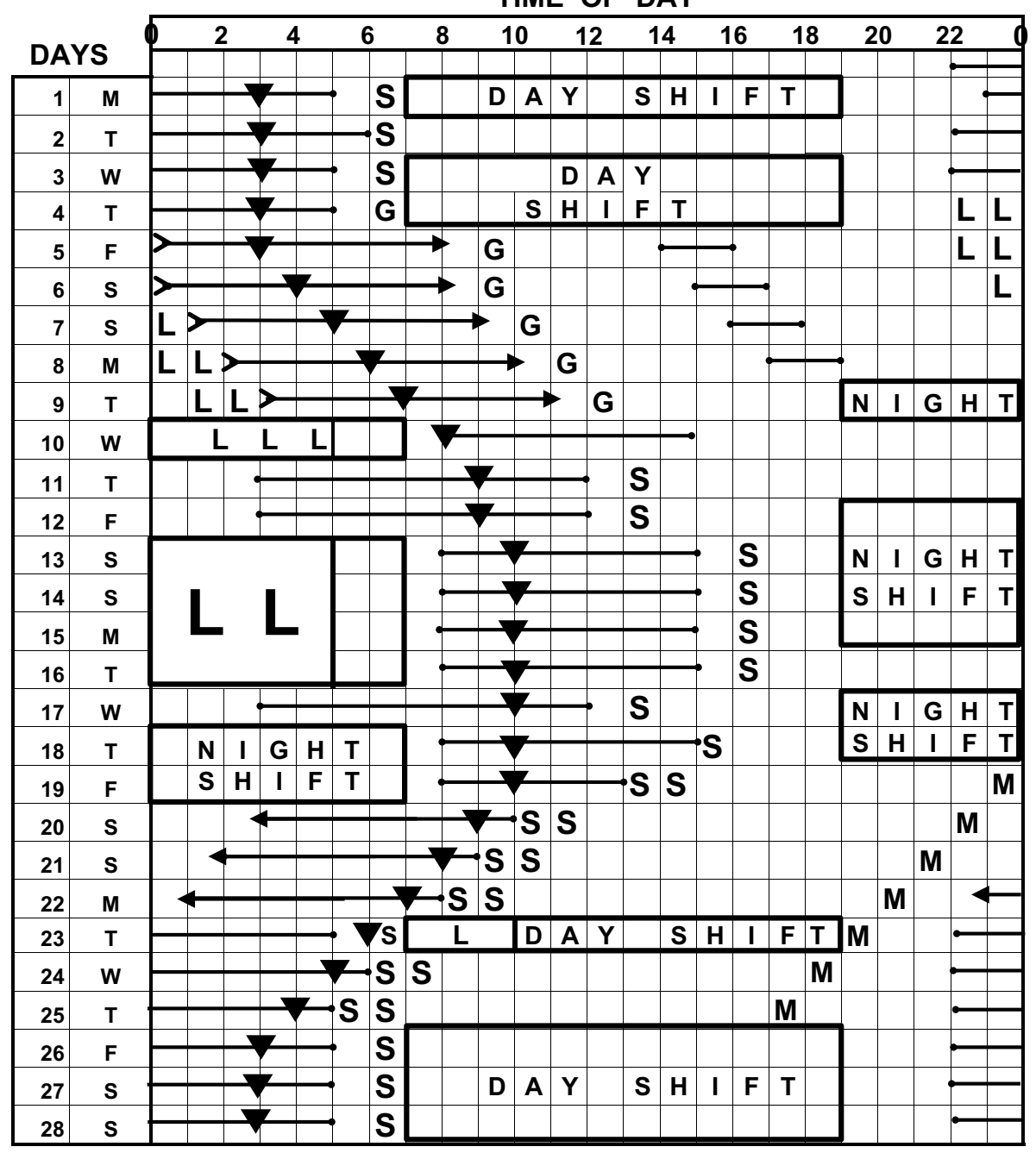

Figure I Light and sleep (dark) schedule for a shift work schedule that rotates between I2-hour day shifts and I2-hour night shifts.

Notes: This schedule is designed to minimize circadian misalignment, improve sleep, improve work performance, and reduce the long-term health risks of shift work. Horizontal lines with dots on both ends - sleep in the dark, short horizontal lines with dots on both ends - naps, sleep (dark) lines with arrow tails and heads pointing to the right - going to bed and waking up a little later is helpful, sleep lines with arrow heads pointing to the left - going to bed earlier is helpful, triangles - the body temperature minimum, a marker for the phase of the circadian clock and the sleepiest time of day. S, sunlight (outdoors); L, bright light from light boxes; M, melatonin (small dose $0.5 \mathrm{mg}$ or I.0 mg).

enough bright light before bed and the avoidance of bright light during sleep and after waking. It is very important to get bright light on these evenings/nights. A light box can be set up on a desk or table next to a computer or laptop or on an end table for watching TV. Breaks from the light box, producing intermittent bright light, are okay. ${ }^{3,25-27}$ Large light boxes are more pleasant to sit near when compared with tiny light boxes that make an aversive bright spot of light ${ }^{28}$ Furthermore, it is easy to inadvertently move out of the range of a small light box. We have shown that the tiny blue GoLITE (Philips CL, Drachten, the Netherlands) can produce phase shifts, but we constantly monitored our subjects to make sure that they were sitting correctly so that the light box was aimed properly. ${ }^{23}$ Very bright white light is not necessary. We have shown that medium intensity light, only a little more intense than ordinary indoor light, can be very effective. ${ }^{29}$
Staying indoors for a few hours after waking on days 5-9 is best, but if going outdoors is necessary, sunglasses can attenuate this morning phase advancing light. We recommend sunglasses that attenuate blue light, often called blue blockers, but that also attenuate the other wavelengths, because all wavelengths can have a phase-shifting effect on the circadian clock. Espresso lenses (Uvex Safety, Smithfield, RI, USA; http://www.uvex.us) are recommended for driving, and we have used them in wrap around Bandit frames (Uvex Safety) in our latest simulated night shift studies. ${ }^{3,18,20}$

On days 10-19, when there are only night shifts and days off, there is an overlap zone for sleep from 8 am to noon; the times for the beginning of sleep after the night shifts and for the end of sleep on days off overlap. The $T_{\min }$ occurs within the overlap zone so that good sleep can be obtained both on days off and after the night shifts. Afternoon outdoor light, 
sunlight (S) on days 11-18, is a light brake to keep the circadian clock from delaying too far.",18 The "Ls" during the night shifts on days 10 and 13-16 indicate intermittent bright light (12 am to $5 \mathrm{am}$ ) from light boxes. This will help delay any workers who have not yet delayed enough from the bright light exposures before bed at home.

To help advance the circadian clock (to make it shift earlier), during the transition from night shifts to day shifts, outdoor light (S) in the mornings on days 19-25 is very important. This morning outdoor sunlight can be intermittent, but the more the better. In bad weather, a light box can be used, but it will not be as powerful as outdoor light even on a cloudy day. The Re-Timer (green light) glasses might also help, but they should not be used while driving. It would also be helpful to have a light box for the beginning of the day shift on day 23 .

A small dose of melatonin $(0.5 \mathrm{mg}$ or $1.0 \mathrm{mg})$ is used in the evenings on days 19-25, around the optimal time to produce a phase advance. ${ }^{30}$ We have shown that a small dose $(0.5 \mathrm{mg})$ does not produce sleepiness when taken in the afternoon, whereas a larger dose $(3.0 \mathrm{mg})$ does have this undesirable side effect. ${ }^{31}$ The effects of appropriately timed light and melatonin for phase advancing are additive, ${ }^{31-33}$ and it is more difficult to advance than to delay the circadian clock, so we recommend using both. Individuals taking prescription medications should check with their doctor before taking melatonin. The arrow heads on the left side of the sleep lines on days 20-22 mean going to bed earlier is helpful.

A schedule similar to days 10-19 containing night shifts and days off has been tested in our laboratory with subjects "working" in the laboratory on performance tests during the night shifts and sleeping at home (Figure 1 in Smith and Eastman $^{3}$ ). As in the current figure, they slept late (3 am to $12 \mathrm{pm}$ ) on days off in between night shifts and went to bed soon after the night shifts. We showed vast improvements in night shift performance and that subjects could sleep within the prescribed sleep times with little deviation. ${ }^{3,20}$ The $T_{\text {min }}$ reached our goal of occurring during the overlap zone in sleep times (ie, during sleep on days off as well as during sleep after night work) after about a week on the schedule.

These subjects started out as day workers and did not use light boxes at home to start their circadian clocks delaying before the start of the night shifts, as in the proposed schedule. Their sleepiest time of day, the $T_{\text {min }}$, started out occurring during the night shifts, as is the case for most real night shift workers, and gradually delayed over the next few days. The proposed schedule makes use of the days off before the first night shift to delay the rhythms so that the $T_{\min }$ never occurs during night work. In our studies, ${ }^{3,20}$ we used intermittent bright light from commercial light boxes that were on for four or five 15-minute intervals during each night shift. In the proposed schedule, intermittent bright light during the night shifts would not have to be so regimented and would not be necessary at all if workers follow the gradually delaying sleep schedule and obtain enough bright light before bed at home on days 4-9.

The light and sleep schedule for permanent night work that we tested in the laboratory $y^{3,18,20}$ and the light and sleep schedule for rotating shift work proposed here are both designed to minimize circadian misalignment, improve sleep, improve night work alertness and performance, and reduce accidents and long-term health risks. They can both reduce melatonin suppression from light at night, which is linked to increased cancer risk, ${ }^{34,35}$ because the melatonin rhythm is shifted into the sleep (dark) episodes, permitting melatonin synthesis. The schedule for permanent night shifts requires light boxes during work, whereas the schedule for rotating shift work mostly requires light boxes at home. The schedule for permanent night work requires the workers to "give up" mornings, because they must go to bed as soon as possible after work and sleep late on days off, whereas the rotating schedule contains several days when workers have free time in the mornings.

The proposed schedule for rotating shift work (Table 2) does not have 7 consecutive days off as in the current schedule for this company (Table 1). It also has a lot of night shifts in a 10-day period, which would be brutal without circadian adaptation (phase shifting). Changing work schedules can require rearranging family responsibilities and other activities that have been scheduled around the work schedule. Thus, it is not surprising that most of these workers and management did not want to change the work schedule. If they had been educated about the detrimental health and safety effects of night shift work, if they were taught basic circadian principles of misalignment and phase shifting, and if the workers could try such a circadian-based schedule on a temporary-only basis and find it to be a great improvement in their lives, then perhaps they would embrace the new schedule. The current schedule (Table 1) probably makes these workers spend the first day or two of those 7 days off recuperating from the 3 grueling weeks of work. The proposed schedule (Table 2) has the advantage of every other weekend off. It is not the most ideal schedule from a circadian rhythm standpoint, but it does meet the scheduling and billing requirements of the company. An even healthier schedule would be to have more than just 2 weeks on one type of shift before having to transition to the next.

Despite decades of laboratory work on how to phase shift the circadian clock, little has been put to use to help real shift workers, with some brave exceptions. ${ }^{36-39}$ Part of the reason may be due to the apparent complexities of using bright light, dark (sleep), and melatonin at specific times. Furthermore, 
the circadian-based schedules discussed here require lifestyle changes by the workers, such as following unusual sleep schedules even on days off. Finally, these recommendations may require employers to change traditional, but unhealthy, shift schedules, and perhaps to furnish light boxes for their workers.

\section{Acknowledgments}

I thank Dean Mikulla for inspiration. The work cited in this paper was supported by NIH grants R01NR007677, R01HL086934, and R01NS23421 and CDC grant R01 OH003954.

\section{Disclosure}

The author reports no conflicts of interest in this work.

\section{References}

1. Akerstedt T. Work hours, sleepiness and the underlying mechanisms. $J$ Sleep Res. 1995;4(suppl 2):15-22.

2. Dinges DF. An overview of sleepiness and accidents. J Sleep Res. 1995;4(suppl 2):4-14.

3. Smith MR, Eastman CI. Shift work: health, performance and safety problems, traditional countermeasures, and innovative management strategies to reduce circadian misalignment. Nat Sci Sleep. 2012;4:111-132.

4. Wright KP Jr, Bogan RK, Wyatt JK. Shift work and the assessment and management of shift work disorder (SWD). Sleep Med Rev. 2012;17(1): $41-54$.

5. Straif K, Baan R, Grosse Y, et al; WHO International Agency For Research on Cancer Monograph Working Group. Carcinogenicity of shift-work, painting, and fire-fighting. Lancet Oncol. 2007;8(12): 1065-1066.

6. Walker J. Frequent alternation of shifts on continuous work. Occup Psychol. 1966;40:215-225.

7. Monk TH. Advantages and disadvantages of rapidly rotating shift schedules-A circadian viewpoint. Hum Factors. 1986;28(5):553-557.

8. Wilkinson RT. How fast should the night shift rotate? Ergonomics. 1992;35(12):1425-1446.

9. Folkard S. Is there a "best compromise" shift system? Ergonomics. 1992; 35(12):1453-1463.

10. Knauth P. The design of shift systems. Ergonomics. 1993;36(1-3):15-28.

11. Chung MH, Kuo TB, Hsu N, Chuo KR, Chu H, Yang CC. Comparison of sleep-related cardiac autonomic function between rotating-shift and permanent night-shift workers. Ind Health. 2011;49(5):589-596.

12. Fernandez RC, Marino JL, Varcoe TJ, et al. Fixed or rotating night shift work undertaken by women: implications for fertility and miscarriage. Semin Reprod Med. 2016;34(2):74-82.

13. Folkard S. Do permanent night workers show circadian adjustment? A review based on the endogenous melatonin rhythm. Chronobiol Int. 2008; 25(2):215-224.

14. Roden M, Koller M, Pirich K, Vierhapper H, Waldhauser F. The circadian melatonin and cortisol secretion pattern in permanent night shift workers. Am J Physiol. 1993;265(1 pt 2):R261-R267.

15. Dumont M, Benhaberou-Brun D, Paquet J. Profile of 24-h light exposure and circadian phase of melatonin secretion in night workers. $J$ Biol Rhythms. 2001;16(5):502-511.

16. Eastman CI, Stewart KT, Mahoney MP, Liu L, Fogg LF. Dark goggles and bright light improve circadian rhythm adaptation to night-shift work. Sleep. 1994;17(6):535-543.

17. Crowley SJ, Lee C, Tseng CY, Fogg LF, Eastman CI. Combinations of bright light, scheduled dark, sunglasses, and melatonin to facilitate circadian entrainment to night shift work. J Biol Rhythms. 2003;18(6): 513-523.
18. Smith MR, Fogg LF, Eastman CI. Practical interventions to promote circadian adaptation to permanent night shift work: study 4. J Biol Rhythms. 2009;24(2):161-172.

19. Crowley SJ, Lee C, Tseng CY, Fogg LF, Eastman CI. Complete or partial circadian re-entrainment improves performance, alertness, and mood during night shift work. Sleep. 2004;27(6):1077-1087.

20. Smith MR, Fogg LF, Eastman CI. A compromise circadian phase position for permanent night work improves mood, fatigue, and performance. Sleep. 2009;32(11):1481-1489.

21. Eastman CI, Martin SK. How to use light and dark to produce circadian adaptation to night shift work. Ann Med. 1999;31(2):87-98.

22. Revell VL, Eastman CI. Jet lag and its prevention. In: Barkoukis TJ, Matheson JK, Ferber R, Doghramji K, editors. Therapy in Sleep Medicine. Philadelphia: Elsevier; 2012:390-401.

23. Revell VL, Molina TA, Eastman CI. Human phase response curve to intermittent blue light using a commercially available device. J Physiol. 2012;590(pt 19):4859-4868.

24. Czeisler CA, Kronauer RE, Allan JS, et al. Bright light induction of strong (type 0) resetting of the human circadian pacemaker. Science. 1989;244(4910):1328-1333.

25. Baehr EK, Fogg LF, Eastman CI. Intermittent bright light and exercise to entrain human circadian rhythms to night work. Am J Physiol. 1999; 277(6 pt 2):R1598-R1604.

26. Rimmer DW, Boivin DB, Shanahan TL, Kronauer RE, Duffy JF, Czeisler CA. Dynamic resetting of the human circadian pacemaker by intermittent bright light. Am J Physiol Regul Integr Comp Physiol. 2000;279(5):R1574-R1579.

27. Gronfier C, Wright KP, Kronauer RE, Jewett ME, Czeisler CA. Efficacy of a single sequence of intermittent bright light pulses for delaying circadian phase in humans. Am J Physiol Endocrinol Metab. 2004;287(1): E174-E181.

28. Eastman CI. How to get a bigger dose of bright light. Sleep. 2011;34(5): 559-560.

29. Martin SK, Eastman CI. Medium-intensity light produces circadian rhythm adaptation to simulated night-shift work. Sleep. 1998;21(2): 154-165.

30. Burgess HJ, Revell VL, Molina TA, Eastman CI. Human phase response curves to three days of daily melatonin: $0.5 \mathrm{mg}$ versus $3.0 \mathrm{mg}$. J Clin Endocrinol Metab. 2010;95(7):3325-3331.

31. Revell VL, Burgess HJ, Gazda CJ, Smith MR, Fogg LF, Eastman CI. Advancing human circadian rhythms with afternoon melatonin and morning intermittent bright light. J Clin Endocr Metab. 2006;91(1): 54-59.

32. Paul MA, Gray GW, Lieberman HR, et al. Phase advance with separate and combined melatonin and light treatment. Psychopharmacology (Berl). 2011;214(2):515-523.

33. BurkeTM, Markwald RR, Chinoy ED, et al. Combination of light and melatonin time cues for phase advancing the human circadian clock. Sleep. 2013;36(11):1617-1624.

34. Stevens RG, Brainard GC, Blask DE, Lockley SW, Motta ME. Breast cancer and circadian disruption from electric lighting in the modern world. CA Cancer J Clin. 2014;64(3):207-218.

35. Schernhammer ES, Schulmeister K. Melatonin and cancer risk: does light at night compromise physiologic cancer protection by lowering serum melatonin levels? Br J Cancer. 2004;90(5):941-943.

36. Boivin DB, James FO. Circadian adaptation to night-shift work by judicious light and darkness exposure. J Biol Rhythms. 2002;17(6):556-567.

37. Stewart KT, Hayes BC, Eastman CI. Light treatment for NASA shiftworkers. Chronobiol Int. 1995;12(2):141-151.

38. Boivin DB, Boudreau P, Tremblay GM. Phototherapy and orange-tinted goggles for night-shift adaptation of police officers on patrol. Chronobiol Int. 2012;29(5):629-640.

39. Stewart KT, Eastman CI. The light stuff: shiftwork, circadian rhythms, and manned spaceflight. In: Holick MF, Jung EG, editors. Biologic Effects of Light 1995. Berlin/New York: Walter de Gruyter \& Co; 1996: $340-347$. 
Dove Medical Press encourages responsible, free and frank academic debate. The content of the ChronoPhysiology and Therapy 'Editorial' section does not necessarily represent the views of Dove Medical Press, its officers, agents, employees, related entities or the ChronoPhysiology and Therapy editors. While all reasonable steps have been taken to confirm the content of each Editorial, Dove Medical Press accepts no liability in respect of the content of any Editorial, nor is it responsible for the content and accuracy of any Editorial.

ChronoPhysiology and Therapy

Dovepress

\section{Publish your work in this journal}

ChronoPhysiology and Therapy is an international, peer-reviewed open access journal focusing on research into the cyclic variations and rhythmicity in physiological processes in the body and the research and development and optimal timing of administration of therapeutic targets to achieve improved outcomes and quality of life for the patient. The manuscript management system is completely online and includes a very quick and fair peer-review system. Visit http://www.dovepress.com/ testimonials.php to read real quotes from published authors. 